Case Report

\title{
Treatment of T3N0 non-small cell lung cancer with chest wall invasion using stereotactic body radiotherapy
}

\author{
William R. Kennedy*, Prashant Gabani, John Nikitas, Pamela P. Samson, Clifford G. Robinson, \\ Jeffrey D. Bradley, Michael C. Roach
}

Department of Radiation Oncology, Washington University School of Medicine, Saint Louis, MO 63110, United States

\section{A R T I C L E I N F O}

\section{Article history:}

Received 9 February 2019

Accepted 19 February 2019

Available online 21 February 2019

\section{Keywords:}

Lung cancer

Chest wall invasion

Stereotactic body radiation therapy

SBRT

T3N0

\begin{abstract}
A B S T R A C T
Objectives: Chest wall invasion (CWI) is observed in $5 \%$ of localized non-small cell lung cancer (NSCLC). The role of stereotactic body radiotherapy (SBRT) in these patients is unknown. We investigate the safety and efficacy of SBRT in patients with T3NO NSCLC due to CWI.

Methods: Patients with T3NO NSCLC due to CWI were identified using a prospective registry. CWI was defined as radiographic evidence of soft tissue invasion or bony destruction. We excluded patients with recurrent or metastatic disease. All patients were treated with definitive SBRT. Prescribed dose was 50 Gy in 5 fractions for most patients. Kaplan-Meier analysis was used to estimate survival outcomes.

Results: We identified 12 patients treated between 2006 and 2017. Median age was 70 (range, 58-85). Median tumor diameter was $3.0 \mathrm{~cm}$ (range, 0.9-7.2). Median survival was 12.0 months (range, 2.4-63). At a median follow-up of 8.9 months (range, 2.1-63), 1-year primary tumor control was $89 \%$, involved lobar control was $89 \%$, local-regional control was $82 \%$, distant control was $91 \%$, and survival was $63 \%$. Of the 4 patients with pre-treatment chest wall pain, 3 reported improvement after SBRT. Two patients reported new grade 1-2 chest wall pain. No grade $3+$ toxicity was reported, with 1 patient experiencing grade 1 skin toxicity and 3 patients experiencing grade 1-2 radiation pneumonitis.

Conclusions: SBRT for CWI NSCLC is safe, with high early tumor control and low treatment-related toxicity. Most patients with pre-treatment chest wall pain experienced relief after SBRT, with no grade 3+ toxicity observed.

(c) 2019 The Authors. Published by Elsevier B.V. on behalf of European Society for Radiotherapy and

Oncology. This is an open access article under the CC BY-NC-ND license (http://creativecommons.org/
\end{abstract} licenses/by-nc-nd/4.0/).

\section{Introduction}

Non-small cell lung cancer (NSCLC) is the leading cause of cancer-related death in both the United States and worldwide [1]. Surgical resection and radiation therapy (RT) are considered standards of care for patients with early-stage NSCLC. For patients with medically-inoperable, early-stage disease, stereotactic body radiotherapy (SBRT) remains standard. Multiple prospective series have shown SBRT achieves high rates of tumor control with acceptably low treatment-related toxicity in both the medically operable and inoperable settings [2-4]. Of patients with localized NSCLC, approximately 5\% have T3N0 staged disease by evidence of chest wall invasion (CWI) at diagnosis [5]. While the Radiation Therapy Oncology Group (RTOG) 0236 trial evaluating SBRT in medically

\footnotetext{
* Corresponding author at: Department of Radiation Oncology, Center for Advanced Medicine, Washington University School of Medicine, 4921 Parkview Place, Lower Level, St. Louis, MO, 63110, United States.

E-mail address: billy.kennedy@wustl.edu (W.R. Kennedy).
}

inoperable early NSCLC did allow patients with T3NO disease, no patients with T3N0 disease were enrolled.

With no prospective data to inform us of the role of SBRT in this select population, questions regarding effectiveness and safety are still yet to be answered. Of particular importance is chest wall toxicity, including skin toxicity, chest wall pain, and rib fractures. While tumors more than $2 \mathrm{~cm}$ from the chest wall or $5 \mathrm{~cm}$ from the posterior skin are at a very low risk, chest wall toxicity is relatively common in tumors of closer proximity [6]. With chest wall toxicity rates ranging from approximately $5 \%$ to $25 \%$, multiple studies have identified additional factors predictive of developing chest wall toxicity, with the volume of irradiated chest wall, tumor size, prescription dose and fractionation all being significant variables [7-9]. However, T3NO NSCLC with CWI commonly presents with pre-treatment chest wall pain and these models did not include this cohort. The purpose of our study is to identify tumor control and tolerability of SBRT in the management of patients with T3NO NSCLC by virtue of CWI. 


\section{Materials and methods}

An institutional review board-approved, prospectivelymaintained database in accordance with the Health Insurance Portability and Accountability Act for patients with newlydiagnosed, previously untreated stage T3NO NSCLC with CWI receiving SBRT with curative intent from 2006 to 2017 was reviewed. All patients signed an informed consent document for inclusion in this database prior to treatment. Chest wall invasion was defined as clinical or radiographic evidence of soft tissue and/or bony involvement, accordingly T3NO per American Joint Committee on Cancer staging, 8th edition [10]. Patients with distant metastatic disease or recurrent disease at time of SBRT were excluded from this analysis. Patients with parietal pleural-based T3N0 lesions without evidence of chest wall soft tissue or bony destruction were also excluded.

All patients were evaluated by a multidisciplinary team, with medical inoperability determined by a cardiothoracic surgeon and/or pulmonologist. Pretreatment evaluation included history and physical examination as well as pulmonary function tests in most patients. All patients were staged via computerized tomography (CT) of the chest, positron emission tomography (PET), and/or mediastinal staging with endobronchial ultrasound (EBUS) or mediastinoscopy for equivocal PET findings. Our institutional SBRT technique has been described in a previous manuscript [11]. Tumor motion was controlled with abdominal compression to minimize the range of tumor motion. Patient setup and target verification was confirmed using daily cone beam CT. Treatment delivery types included noncoplanar 3-dimensional conformal (3DCRT), coplanar intensity-modulated radiation therapy (IMRT), and coplanar volumetric modulated arc therapy (VMAT). For 3DCRT, prescription was typically to the $75 \%$ to $85 \%$ isodose line so that $95 \%$ of the prescribed dose covers the PTV. For IMRT/VMAT treatments, plan optimization was set to recapitulate similar hotspots as the 3DCRT plans. No specific chest wall constraints were utilized in the treatment planning process, although hotspots are typically avoided in the chest wall, if possible. For our five-fraction scheme, normal tissue constraints were respected for the spinal cord (maximum dose (Dmax)<20 Gy), esophagus (Dmax <30 Gy), heart (Dmax $<30 \mathrm{~Gy}$ ), brachial plexus (Dmax $<25 \mathrm{~Gy}$ ), and the volume of lung minus internal target volume (ITV) receiving at least 20 Gy (V20Gy) < 10\%.

Patients were followed with serial CT scans at 6 weeks' posttreatment, then typically every 3 months for 2 years, followed by every 6 months for an additional 3 years. Survival was calculated from time of treatment until death or last follow-up. Primary tumor failure was defined as radiographic evidence of local enlargement of the treated primary tumor. Hilar and mediastinal nodal recurrences were included in local-regional failures. The Kaplan-Meier method was used to estimate control and survival probabilities and Cox analyses were performed. Significance was considered at $\mathrm{p}<0.05$ and all significance levels were 2-sided. IBM $^{\circledR}$ SPSS $^{\circledR}$ Statistics, version 23 was applied for all statistical analyses.

\section{Results}

\subsection{Patients and treatment characteristics}

We identified 12 patients who met inclusion and exclusion criteria. Patient, tumor, and treatment-related characteristics are summarized in Table 1 . Median age at diagnosis was 70 years (range, 58-85). No patients had received prior in-field RT or previous chemotherapy. Prescription dose was $50 \mathrm{~Gy}$ in 5 fractions (biological effective dose, $\mathrm{BED}_{10}=100 \mathrm{~Gy}$ ) for all but 3 patients: two
Table 1

Patient- and treatment-related characteristics $(n=12)$.

\begin{tabular}{|c|c|}
\hline Characteristic & Value (\% or range) \\
\hline Age (years), median & $70(58-85)$ \\
\hline \multicolumn{2}{|l|}{ Sex } \\
\hline Male & $5(42 \%)$ \\
\hline Female & $7(58 \%)$ \\
\hline BMI, median & $24.6(17.3-40)$ \\
\hline \multicolumn{2}{|l|}{ Smoking status } \\
\hline Never & $0(0 \%)$ \\
\hline Former & $6(50 \%)$ \\
\hline Current & $6(50 \%)$ \\
\hline Smoking pack-years & $65(15-108)$ \\
\hline Age-adjusted CCI, median & $5(3-10)$ \\
\hline KPS, median & $60(50-80)$ \\
\hline Pretreatment hemoglobin (g/dl), mean & $12.7(9.8-15.7)$ \\
\hline \multicolumn{2}{|l|}{ Histology } \\
\hline Squamous cell carcinoma & $6(50 \%)$ \\
\hline Adenocarcinoma & $2(17 \%)$ \\
\hline NSCLC NOS & $2(17 \%)$ \\
\hline Biopsy unable to be obtained & $2(17 \%)$ \\
\hline Tumor diameter $(\mathrm{cm})$, median & $2.7(0.9-7.2)$ \\
\hline PET SUV, median & $13.8(4.4-25.9)$ \\
\hline \multicolumn{2}{|l|}{ Mediastinal staging } \\
\hline PET alone & $8(66 \%)$ \\
\hline EBUS & $2(17 \%)$ \\
\hline Mediastinoscopy & $2(17 \%)$ \\
\hline \multicolumn{2}{|l|}{ Dose and fractionation } \\
\hline $50 \mathrm{~Gy}$ in 5 fractions & $9(75 \%)$ \\
\hline $54 \mathrm{~Gy}$ in 3 fractions & $2(17 \%)$ \\
\hline $62.5 \mathrm{~Gy}$ in 10 fractions & $1(8 \%)$ \\
\hline \multicolumn{2}{|l|}{ Treatment schedule } \\
\hline Consecutive days & $2(17 \%)$ \\
\hline Nonconsecutive days & $10(83 \%)$ \\
\hline \multicolumn{2}{|l|}{ Delivery Type } \\
\hline Noncoplanar 3D conformal & $9(75 \%)$ \\
\hline Coplanar IMRT & $2(17 \%)$ \\
\hline Noncoplanar VMAT & $1(8 \%$ \\
\hline PTV volume $\left(\mathrm{cm}^{3}\right)$, mean & $72.2(10.8-210.6)$ \\
\hline
\end{tabular}

BMI, body mass index; CCI, Charlson comorbidity index; KPS, Karnofsky performance status, NSCLC NOS, non-small cell lung cancer not otherwise specific; PET SUV, positron emission tomography standardized uptake value; EBUS, endobronchial ultrasound; IMRT, intensity-modulated radiation therapy; VMAT, volumetric modulated arc therapy; PTV, planning target volume

received $54 \mathrm{~Gy}$ in 3 fractions $\left(\mathrm{BED}_{10}=151 \mathrm{~Gy}\right)$ and one other patient received $62.5 \mathrm{~Gy}$ in 10 fractions $\left(\mathrm{BED}_{10}=102 \mathrm{~Gy}\right)$. Patients received treatment every other day (10 patients) with the remaining two receiving fractions on consecutive week days. For the 9 patients requiring motion control, abdominal compression was used in all but 1 patient, who was managed with BodyFix (Bodyfix; Medical Intelligence, Inc., Quebec, QC, Canada) vacuum restriction bag. The remaining 3 patients had minimal tumor motion so compression was not used. Delivery was predominantly with noncoplanar 3DCRT (9 patients), with 2 patients being treated with coplanar IMRT and 1 other patient with noncoplanar VMAT (Fig. 1). No patients received adjuvant chemotherapy. Pretreatment pulmonary function tests were obtained in 8 patients (66\%). The median FEV1 was $1.3 \mathrm{~L}$ and $44 \%$ (range, $0.4-2.5 \mathrm{~L}$ and $22-72 \%$, respectively), and median DLCO was $46 \%$ (range, $33-$ $69 \%$ ). Only 2 patients had post-treatment testing, however, with minimal changes observed in both.

\subsection{Outcomes}

The median follow-up for all patients was 8.9 months (range, 2.1-62.5 months). The median overall survival was 12.0 months (2.4-62.8 months). For surviving patients, the estimated 1-year primary tumor control was $89 \%$, involved lobar control was $89 \%$, local-regional control was $82 \%$, distant control was $91 \%$, and over- 


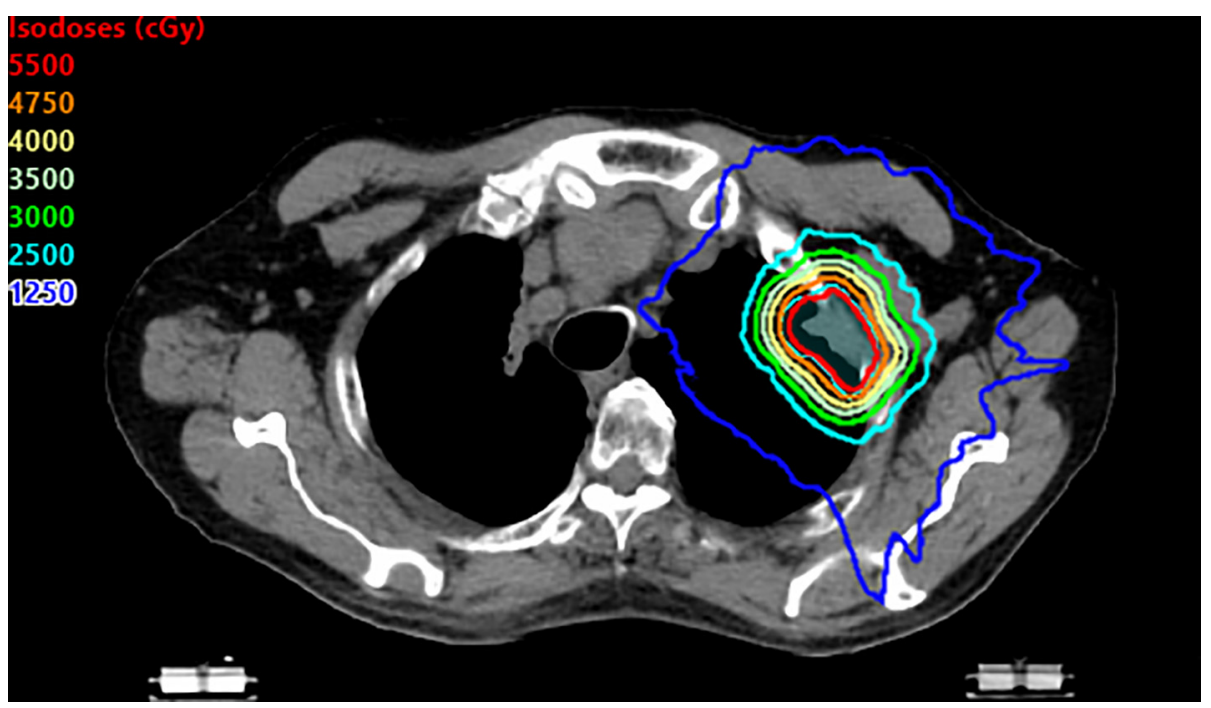

Fig. 1. Prescription isodose distributions for a patient treated to $50 \mathrm{~Gy}$ in 5 fractions using noncoplanar VMAT.
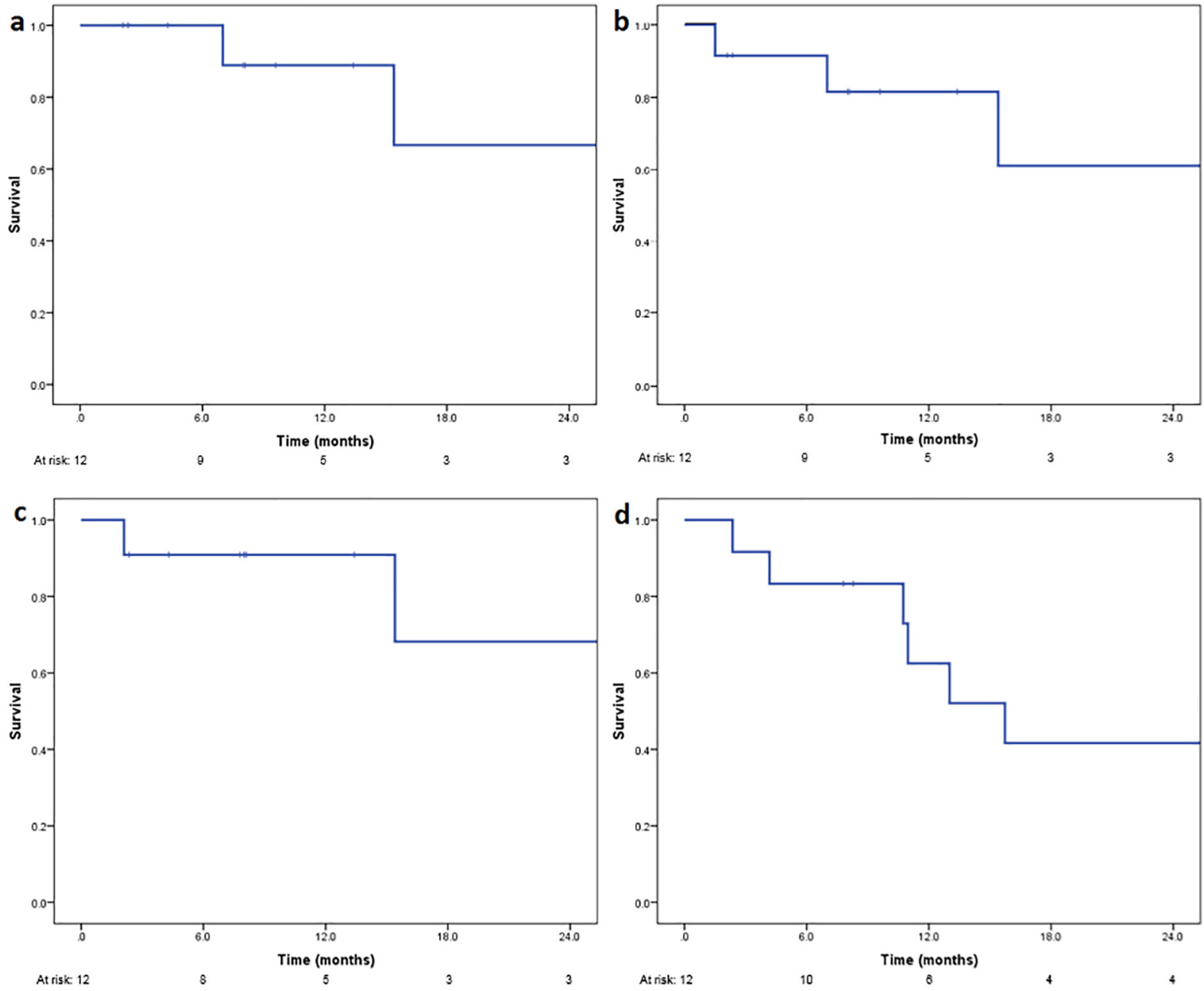

Fig. 2. Kaplan-Meier curves for local control (a), nodal control (b), distant control (c), and overall survival (d).

all survival was $63 \%$ (Fig. 2). Table 2 shows the patterns of failure among study patients. Nodal and distant failures were the most common sites of treatment failure. No isolated primary tumor fail- ures were observed. Of the two patients who experienced local failures, both had either nodal or distant involvement at time of local failure as well. 
Table 2

Patterns of failure among study patients $(n=12)$.

\begin{tabular}{ll}
\hline Failure type & Number \\
\hline Local & 2 \\
Local only & 0 \\
Local and nodal & 1 \\
Local, lobar, nodal and distant & 1 \\
Nodal & 4 \\
Nodal only & 1 \\
Nodal and distant & 1 \\
Distant & 4 \\
Distant only & 2 \\
Metachronous lung & 0 \\
\hline
\end{tabular}

Of the 4 patients who presented with pre-treatment chest wall pain, 3 (75\%) of them reported either improvement or resolution of their symptoms, with one patient reporting improvement but not resolution of their symptoms with no further need for narcotic use, and the other two patients reporting complete resolution of their symptoms. The other patient reported no change in the symptoms post-treatment. Of the 8 patients who did not have pre-treatment chest wall pain, 2 patients developed chest wall pain requiring over-the-counter medication. The first patient developed chest wall pain 2 months after SBRT, correlating with development of pleural-based metastases outside the treatment field. This patient's symptoms were improved with over-thecounter non-steroidal anti-inflammatory (NSAID) medication, and the patient passed away from metastatic disease before resolution of their symptoms was documented. The other patient developed mild rib pain 13 months after treatment that did not require narcotics and resolved with a short course of NSAIDs. No patients developed treatment-related chest wall pain requiring narcotics after SBRT and no patients had clinical or radiographic evidence of rib fractures on follow-up CT imaging. Grade 1 skin toxicity was observed in 1 patient after treatment which resolved spontaneously. Radiation pneumonitis was observed in 3 patients, grade 1 in two cases and only one case of symptomatic grade 2 pneumonitis managed with steroids. No grade $3+$ pneumonitis or other toxicity was observed in this cohort.

\section{Discussion}

We report that SBRT can be safely and effectively delivered in patients with primary T3NO NSCLC invading the chest wall. The frequency of treatment-associated toxicity was low, with no grade $3+$ events and symptoms typically being mild and/or self-limited in the few patients experiencing side effects. Local and lobar control were high at $89 \%$ each in our cohort, although duration of follow-up was limited, with nodal and distant spread being the most common patterns of failure in this medically high-risk patient population. Importantly, in the subset of patients presenting with pre-treatment chest wall pain, most patients experienced relief of their symptoms, indicating that SBRT may be an effective tool to provide pain relief by virtue of local control.

Historically, patients with lung cancer invading the chest wall have presented unique challenges to definitive management. The standard of care for these patients has long been en bloc surgical resection, often followed by adjuvant chemotherapy. Generally, acceptable margins are $1 \mathrm{~cm}$ in all directions, although some argue for removing 1 rib above and below the tumor as well as achieving lateral margins of 3 to $4 \mathrm{~cm}$, given that survival is highly dependent on completeness of resection [12]. The necessity of complete resection is balanced by the fact that these are often extensive surgeries requiring reconstruction of large chest wall defects, although chest wall defects less than $5 \mathrm{~cm}$ can typically be managed without reconstruction [5]. Even at high-volume centers with skilled thoracic surgeons, perioperative mortality is relatively high, with experiences from Memorial Sloan-Kettering and multi-center French series reporting mortality rates of up to $8 \%$ [13-15]. Survival after surgical resection varies significantly in the literature but is generally poor, as 5-year overall survival is approximately $40 \%$, with margin status, extent of invasion, and pathologic nodal status all significantly influencing this rate [16-18].

Given that surgical resection presents unique, significant challenges and that many patients are poor operative candidates due to comorbid cardiopulmonary disease, nonoperative approaches to management are often necessary. Conventionally-fractionated radiotherapy has long been described in medically-inoperable NSCLC, with 2-year disease-free survival only $25 \%$ for T3NO patients treated to doses of at least $60 \mathrm{~Gy}$ in $2 \mathrm{~Gy}$ fractions [19]. Given historically poor disease control rates with conventionally fractionated definitive radiotherapy alone for early stage NSCLC as well as the medical frailty of this demographic, it is unsurprising that survival at 2- and 5-years has been 35\% and 15\%, respectively, in multiple large series $[20,21]$.

Chest wall pain is a common presenting symptom in these patients, causing significant detriment to quality of life and further underscoring the importance of local control as an important metric in this cohort. Accordingly, the local control in our series was $89 \%$ at 1 year, comparable to reported rates in large SBRT series of T1-2N0 lesions not invading the chest wall [22,23]. The need for maximizing local control is balanced with minimizing treatment-related chest wall toxicity, as large SBRT series have shown rates of acute and chronic chest wall pain of approximately $5 \%$ and $17 \%$, respectively with chest wall V30 and obesity predicting for both pain and skin toxicity [24]. In our subset of patients that did present with pre-treatment chest wall pain, $75 \%$ achieved pain relief with $50 \%$ experiencing complete pain relief. These findings corroborate the Cleveland Clinic experience with treating 10 patients with primary T3NO NSCLC and an additional 3 patients with recurrent NSCLC invading the chest wall, which showed pain relief of $80 \%$, with $50 \%$ achieving complete resolution of symptoms [25]. To our knowledge, there are no other published studies todate characterizing outcomes other than the above work by Cleveland Clinic and our current series. While two of our patients did experience new chest wall pain after treatment, both cases were grade 1-2 with one of these likely due to development of pleural-based metastases and the other relieved with over-thecounter medication. We observed no grade $3+$ chest wall toxicity, including no rib fractures, although these events may be observed over 1-year post-treatment. Furthermore, although post-SBRT pulmonary function testing was only obtained in 2 of our patients, no clinically-significant changes from baseline were observed, consistent with other series [26].

Nodal and distant relapse were much more common than local failures in our series, which is consistent with the greater SBRT experience for early-stage NSCLC [27]. This observation has led some to advocate for adjuvant chemotherapy after SBRT, extrapolating from the survival advantage seen in the Cancer and Leukemia Group B (CALGB) 9633 trial for node-negative tumors $4 \mathrm{~cm}$ or greater who had undergone adjuvant chemotherapy after surgery, although none of our patients with larger tumors received adjuvant systemic therapy [28]. Following a similar logic, multiple retrospective series have shown improved survival with adjuvant chemotherapy in surgically-resected pT3NO NSCLC with chest wall invasion, independent of size [29-31]. In our series, one third of patients developed regional nodal metastases as a component of failure, which might be decreased by systemic therapy. Despite these observations, the role of adjuvant systemic therapy after SBRT for NSCLC is still poorly-defined and may warrant prospective investigation. 
Our study is affected by several limitations inherent to its design. We investigated a small population of patients with multiple medical comorbidities, with follow-up duration limited by the high number of intercurrent deaths in the study interval. Consequently, estimates of tumor control are short and further followup with larger sample size may provide more accurate assessment of these measures. Rates of treatment-related toxicity may be underestimated by retrospective review, and the addition of patient-reported quality of life data would further delineate the analgesic effect of SBRT in this cohort. Furthermore, SBRT techniques have evolved over the study duration at our institution. Currently, patients are typically treated on consecutive days while most of the patients in our study were treated when treatment on nonconsecutive days was routine at our institution, although previous studies have shown no impact of treatment schedule on outcome [32]. Furthermore, while most patients on our study were treated with noncoplanar 3D conformal techniques, we are increasingly treating patients with VMAT, although its impact on control and toxicity, if any, are unknown. Despite these limitations, SBRT appears to be a promising treatment modality for patients with chest wall-invading tumors.

\section{Conclusion}

SBRT for patients with NSCLC invading the chest wall appears to be a feasible, safe, and well-tolerated treatment modality associated with high rates of early tumor control. For patients experiencing local symptoms, SBRT is effective in providing at least partial pain relief. For medically-inoperable patients with T3NO NSCLC due to CWI, SBRT should be considered as a potential management option, and warrants further investigation.

\section{Declarations}

Ethics approval and consent: Approved by the institutional review board in accordance with the Health Insurance Portability and Accountability Act.

Consent for publication: Not applicable.

Availability of data and materials: Please contact author for data requests

Authors' contributions: WK was responsible for study conception and design, acquisition of data, analysis and interpretation of data, and drafting of the manuscript. PG was responsible for acquisition of data, analysis and interpretation of data. JN was responsible for acquisition of data. CR and JB were responsible for critical revision. MR was responsible for study conception and design, interpretation of data, and critical revision.

\section{Funding}

Supported by Clinical and Translational Science Award (CTSA) Grant [UL1 TR000448] and Siteman Comprehensive Cancer Center and NCI Cancer Center Support Grant P30 CA091842.

\section{Conflict of interest}

We have no conflict of interest to disclose for this work.

\section{Acknowledgements}

Not applicable.

\section{Appendix A. Supplementary data}

Supplementary data to this article can be found online at https://doi.org/10.1016/j.ctro.2019.02.004.

\section{References}

[1] Torre LA, Siegel RL, Jemal A. Lung cancer statistics. Adv Exp Med Biol 2016;893:1-19.

[2] Timmerman RD, Hu C, Michalski JM, et al. Long-term results of stereotactic body radiation therapy in medically inoperable stage i non-small cell lung cancer. JAMA Oncol 2018;4:1287-8.

[3] Chang JY, Senan S, Paul MA, et al. Stereotactic ablative radiotherapy versus lobectomy for operable stage I non-small-cell lung cancer: a pooled analysis of two randomised trials. Lancet Oncol 2015;16:630-7.

[4] Timmerman RD, Paulus R, Pass HI, et al. stereotactic body radiation therapy for operable early-stage lung cancer: findings from the NRG oncology RTOG 0618 trial. JAMA Oncol 2018;4:1263-6.

[5] Lanuti M. Surgical management of lung cancer involving the chest wall. Thorac Surg Clin 2017:27:195-9.

[6] Stephans KL, Djemil T, Tendulkar RD, et al. Prediction of chest wall toxicity from lung stereotactic body radiotherapy (SBRT). Int J Rad Oncol*Biol*Phys 2012;82:974-80.

[7] Dunlap NE, Cai J, Biedermann GB, Wall Chest, et al. Volume receiving $>30$ Gy predicts risk of severe pain and/or rib fracture after lung stereotactic body radiotherapy. Int J Rad Oncol*Biol*Phys 2010;76:796-801.

[8] Mutter RW, Liu F, Abreu A, et al. Dose-volume parameters predict for the development of chest wall pain after stereotactic body radiation for lung cancer. Int J Radiat Oncol Biol Phys 2012;82:1783-90.

[9] Woody NM, Videtic GMM, Stephans KL, et al. Predicting chest wall pain from lung stereotactic body radiotherapy for different fractionation schemes. Int J Rad Oncol*Biol*Phys 2012;83:427-34.

[10] Rami-Porta R, Bolejack V, Giroux DJ, et al. The IASLC lung cancer staging project: the new database to inform the eighth edition of the TNM classification of lung cancer. J Thorac Oncol 2014;9:1618-24.

[11] Bradley JD, El Naqa I, Drzymala RE, et al. Stereotactic body radiation therapy for early-stage non-small-cell lung cancer: the pattern of failure is distant. Int J Rad Oncol*Biol*Phys 2010;77:1146-50.

[12] Facciolo F, Cardillo G, Lopergolo M, et al. Chest wall invasion in non-small cell lung carcinoma: a rationale for en bloc resection. J Thorac Cardiovasc Surg 2001:121:649-56.

[13] Downey RJ, Martini N, Rusch VW, et al. Extent of chest wall invasion and survival in patients with lung cancer. Ann Thorac Surg 1999;68:188-93.

[14] Magdeleinat P, Alifano M, Benbrahem C, et al. Surgical treatment of lung cancer invading the chest wall: results and prognostic factors. Ann Thorac Surg 2001;71:1094-9.

[15] Doddoli C, D'Journo B, Pimpec-Barthes FL, et al. Lung cancer invading the chest wall: a plea for en-bloc resection but the need for new treatment strategies. Ann Thorac Surg 2005;80:2032-40.

[16] Chapelier A, Fadel E, Macchiarini P, et al. Factors affecting long-term survival after en-bloc resection of lung cancer invading the chest wall. Eur J Cardiothorac Surg 2000;18:513-8.

[17] Voltolini L, Rapicetta C, Luzzi L, et al. Lung cancer with chest wall involvement: predictive factors of long-term survival after surgical resection. Lung Cancer 2006:52:359-64.

[18] Piehler JM, Pairolero PC, Weiland LH, et al. Bronchogenic carcinoma with chest wall invasion: factors affecting survival following en bloc resection. Ann Thorac Surg 1982;34:684-91.

[19] Dosoretz DE, Katin MJ, Blitzer PH, et al. Radiation therapy in the management of medically inoperable carcinoma of the lung: results and implications for future treatment strategies. Int J Radiat Oncol Biol Phys 1992;24:3-9.

[20] Graham PH, Gebski VJ, Stat M, et al. Radical radiotherapy for early nonsmall cell lung cancer. Int J Rad Oncol*Biol*Phys 1995;31:261-6.

[21] Sibley GS, Jamieson TA, Marks LB, et al. Radiotherapy alone for medically inoperable stage I non-small-cell lung cancer: the duke experience. Int J Rad Oncol*Biol*Phys 1998;40:149-54.

[22] Onishi H, Shirato H, Nagata Y, et al. Hypofractionated stereotactic radiotherapy (HypoFXSRT) for stage I non-small cell lung cancer: updated results of 257 patients in a Japanese multi-institutional study. J Thorac Oncol 2007;2: S94-100.

[23] Murray P, Franks K, Hanna GG. A systematic review of outcomes following stereotactic ablative radiotherapy in the treatment of early-stage primary lung cancer. Br J Radiol 2017;90.

[24] Welsh J, Thomas J, Shah D, et al. Obesity increases the risk of chest wall pain from thoracic stereotactic body radiation therapy. Int J Radiat Oncol Biol Phys 2011;81:91-6.

[25] Berriochoa C, Videtic GMM, Woody NM, et al. Stereotactic body radiotherapy for T3N0 lung cancer with chest wall invasion. Clin Lung Cancer 2016;17:595-601. 
[26] Stanic S, Paulus R, Timmerman RD, et al. No clinically significant changes in pulmonary function following stereotactic body radiation therapy for earlystage peripheral non-small cell lung cancer: an analysis of RTOG 0236. Int J Radiat Oncol Biol Phys 2014;88:1092-9.

[27] Chi A, Liao Z, Nguyen NP, et al. Systemic review of the patterns of failure following stereotactic body radiation therapy in early-stage non-small-cell lung cancer: Clinical implications. Radiother Oncol 2010;94:1-11.

[28] Strauss GM, Herndon JE, Maddaus MA, et al. Adjuvant paclitaxel plus carboplatin compared with observation in stage IB non-small-cell lung cancer: CALGB 9633 with the cancer and leukemia group B, radiation therapy oncology group, and north central cancer treatment group study groups. JCO 2008;26:5043-51.
[29] Ahmad U, Crabtree TD, Patel AP, et al. Adjuvant chemotherapy is associated with improved survival in locally invasive node negative non-small cell lung cancer. Ann Thorac Surg 2017;104:303-7.

[30] Gao SJ, Corso CD, Blasberg JD, et al. Role of adjuvant therapy for nodenegative lung cancer invading the chest wall. Clin Lung Cancer 2017; 18(169-177):e4

[31] Drake JA, Sullivan JL, Weksler B. Adjuvant chemotherapy improves survival in patients with completely resected T3NO non-small cell lung cancer invading the chest wall. J Thorac Cardiovasc Surg 2018;155:1794-802.

[32] Samson P, Rehman S, Juloori A, et al. Local control for clinical stage I non-small cell lung cancer treated with 5 -fraction stereotactic body radiation therapy is not associated with treatment schedule. Pract Rad Oncol 2018. 